des Begriffs in den Vordergrund und andererseits wurde deutlich, dass Intersektionalität keine eigenständige Theorie oder Methode im Sinne eines Regelwerks ist, sondern eher eine methodologische, heuristische, theoretische Haltung darstellt. Hierüber muss weiter debattiert werden.
Alle, die sich näher mit den einzelnen Vorträgen befassen möchten, können sich freuen: Die Beiträge der Konferenz werden auf der Homepage unter der folgenden URL veröffentlicht: http://www. cgc.uni-frankfurt.de/intersectionality/index.shtml

\title{
Viktorija Ratković
}

\section{„Das erste Opfer des Krieges ist die ... Emanzipation. Zum Zusammenhang von Medien, Krieg und Geschlecht«}

Tagung der DGPuK-Fachgruppe Medien, Öffentlichkeit und Geschlecht und des Fachbereichs Kommunikationswissenschaft der Universität Salzburg in Salzburg am 2. und 3. Oktober 2008

Veranstaltet wurde die Tagung "Das erste Opfer des Krieges ist die ... Emanzipation" durch die Fachgruppe Medien, Öffentlichkeit und Geschlecht der Deutschen Gesellschaft für Publizistik und Kommunikationswissenschaft (Martina Thiele, Tanja Thomas) und den Fachbereich Kommunikationswissenschaft der Universität Salzburg (Elisabeth Klaus) in Kooperation mit dem Institut für Medien- und Kommunikationswissenschaft der Alpen-Adria-Universität Klagenfurt (Brigitte Hipfl) sowie dem Zentrum für Konfliktforschung an der Philipps-Universität Marburg (Fabian Virchow).

In ihrer Einführung zu "Medien, Krieg und Geschlecht" ging Tanja Thomas auf den von den VeranstalterInnen bewusst provokant formulierten Titel der Tagung ein und erklärte, dass diese unter "Emanzipation" jeden Kampf um Emanzipation verstehen und ihn nicht lediglich in Bezug zu Frauen setzen. Sie formulierte auch die Frage, die bei der Tagung beantwortet werden sollte, nämlich, ob und wie $\mathrm{Me}-$ dien im Krieg bzw. Krieg in den Medien Emanzipationsprozesse eröffnen (können).
"Militär und Geschlecht" kristallisierte sich im Laufe der Tagung als einer der bestimmenden Themenbereiche heraus. In ihrer Keynote "Gender, Militär und Krieg: Komparative Perspektiven" führte Christine Eifler (Bremen) aus, dass Soldatinnen Zuschreibungen von Geschlechteridentitäten in Frage stellen, indem sie die institutionelle Verknüpfung von Männlichkeit und Gewalt durchbrechen. Auch leitet der Einsatz von Frauen im Militär einen Prozess der Zivilisierung des Militärs ein, welcher Auswirkungen auf die gesamte Gesellschaft hat, indem sich auch auf symbolischer Ebene Verknüpfungen von Militär und Geschlecht ändern.

In ihren Medienanalysen zur Darstellung von Soldatinnen arbeiteten sowohl Susanne A. Friedel (Berlin) als auch Susanne Kassel (Salzburg) heraus, dass in vom Militär herausgegebenen Medien (in Deutschland und Israel) stereotype $\mathrm{Zu}$ schreibungen an Soldatinnen als Frauen vorherrschen, indem diese beispielsweise als "Zierde der Einheit" portraitiert werden. Friedel fuihrte auch aus, dass der Militärdienst in Israel nicht zur Hinterfragung 
der vorhandenen Geschlechterrollen führt, sondern dass den Soldatinnen vielmehr laufend ihre eigene Weiblichkeit bewusst gemacht wird, indem ihnen sowohl ihre fehlende Männlichkeit als auch die angeblich fehlende physische Eignung vorgefuihrt werden. Als Ergebnis seiner Analyse der Darstellung von Soldaten in aktuellen Filmen präsentierte Marcus S. Kleiner (Bonn) das, in den analysierten Filmen herrschende, Bild des "neuen " Soldaten, der zwar überVerständnis für fremde Kulturen verfügt, Gutes tun will usw., dessen Weltund Selbstentwurf sich aber nicht gegenüber früher verändert haben. Mit dem Themenkomplex "Medien, Militär und Geschlecht" setzte sich auch Saskia Stachowitsch (Wien) auseinander, wobei ihr Fokus auf "Mediale(n) Geschlechterideologien als Folge ziviler und militärischer Arbeitsmarktentwicklung in den USA" lag. Sie hielt fest, dass Medien zu Kriegszeiten und bei Rekrutierungsproblemen positiver über Soldatinnen berichten als in Zeiten, in denen kein Rekrutierungsproblem herrscht.

Historische Bezüge bildeten ebenfalls einen roten Faden der Tagung, wobei gerade der Erste Weltkrieg Eingang in einige Vorträge fand. Anhand von Biografien von Journalistinnen im Ersten Weltkrieg fuihrten Elisabeth Klaus (Salzburg) und Ulla Wischermann (Frankfurt) vor, dass kein Kausalzusammenhang zwischen Feminismus und Pazifismus besteht. Auf die mediale Repräsentation von Frauen in Medien zur Zeit des Ersten Weltkrieges fokussierten sich Eva Krivanec (Wien) in ihrem Vortrag "Unterhaltungstheater als Medium der Verhandlung von Geschlechterrollen im Krieg" und Susanne A. Fehlmann (Düsseldorf), die die Darstellung von Soldatenmüttern thematisierte. Hanne Hieber (Dortmund) stellte die Frage "Emanzipation im Geheimdienst?" und referierte über Elsabeth Schragmüller, die im Ersten Weltkrieg die Leiterin der
Spionageabteilung Frankreich des deutschen Geheimdienstes war und die u.a. als "Mademoiselle Docteur" zur Legende geworden ist. In seinem Vortrag "Patriotische Frauen gegen den Irak-Krieg" beleuchtete Jörg-Uwe Nieland (Duisburg) den Krieg aus heutiger Perspektive, wobei er am Beispiel der texanischen (Country-)Band Dixie Chicks verschiedene Weiblichkeitskonzepte des Protests aufzeigte und auf die gesellschaftskritische Prägekraft der politischen Popmusik einging.

Die Auseinandersetzung mit Stereotypen bildete einen weiteren wichtigen Schwerpunkt der Tagung. Wiewohl sie unterschiedliche Medien und Fokussierungen wählten, glichen sich die Ergebnisse der Analysen von Martina Thiele (Salzburg), Tanja Maier (Siegen) und Anna Bergmann (Berlin): So nehmen in Kriegszeiten Stereotype - sowohl was Frauen als auch Männer betrifft - insgesamt zu, gleichzeitig werden alte Geschlechterbilder in neue Kontexte gesetzt. Westliche Medien greifen dabei laufend auf die im europäischen kulturellen Gedächtnis verankerten stereotypen Geschlechterbilder zurück. Fabian Virchow (Marburg) und Tanja Thomas (Lüneburg) führten aus, dass in Berichten über Selbstmordattentäterinnen keine Verschiebung von stereotypen Geschlechterrollen stattfindet, sondern dass den Frauen vielmehr ihr Subjektstatus abgesprochen wird, indem ihnen beispielsweise keine politischen, sondern vielmehr private Motive (etwa eine Wiederherstellung der Ehre) unterstellt werden.

In einer abschließenden Podiumsdiskussion gingen die Journalistinnen Andrea Böhm (Die Zeit), Sibylle Hamann (Falter, Die Presse), Gudrun Harrer (Der Standard), Rubina Möhring (Reporter ohne Grenzen) und Ute Scheub (taz) auf die Frage ein, welche Rolle das Geschlecht von JournalistInnen in der (Kriegs-)Berichterstattung spielt. Sie stellten fest, dass es einerseits von Vorteil sein kann, 
eine Frau $\mathrm{zu}$ sein (beispielsweise haben nur Journalistinnen Kontakte zu Frauen in Afghanistan), andererseits werden in muslimischen Ländern gerade weibliche JournalistInnen als Repräsentantinnen des vermeintlich feindlichen Westens wahrgenommen und sind deswegen $z$.T. persönlichen Anfeindungen ausgesetzt. Diskutiert wurde ebenfalls die Frage, wie JournalistInnen über Gewalttätigkeit berichten können, ohne Stereotype zu verwenden, wobei die geäußerte Vorstellung, dass es möglich sei, Ereignisse so zu beschreiben, "wie sie sind" von den TeilnehmerInnen der Tagung stark hinterfragt wurde. Betont wurde die Notwendigkeit der Reflexion; so wurde die Tatsache, dass Journalistinnen bei der Tagung "Das erste Opfer des Krieges ist die ... Emanzipation" die Mög- lichkeit der Teilnahme und Diskussion geboten wurde, als sehr positiv bewertet.

In ihrem Schlusswort ging Elisabeth Klaus (Salzburg) auf mögliche Erklärungen fuir die Konzentration der Vortragenden auf historische Rückblicke und den Themenkomplex Militär und Geschlecht ein. So haben historische Rückblicke den Vorteil, dass sie aufzeigen können, inwieweit aktuelle Entwicklungen tatsächlich neu sind oder sich vielmehr auf Altes beziehen. Die Thematisierung von Militär und Geschlecht erscheint vor dem Hintergrund der vermehrten Zivilisierung des Militärs sehr aktuell, wobei Elisabeth Klaus die Frage aufwarf, inwieweit diese auch die Gefahr der Militarisierung der Gesellschaft enthält, etwa durch das Eindringen militärischer Themen in den Alltag.

\section{Heidi Degethoff de Campos, Marianne Kriszio}

\section{Mittendrin statt nur dabei. 100 Jahre Frauenstudium in Preußen}

\section{Festveranstaltung am 24. Oktober 2008 im Roten Rathaus in Berlin}

Anlässlich des 100jährigen Jubiläums der offiziellen Zulassung von Frauen zum Studium an der Berliner Universität und im übrigen Preußen organisierte die Arbeitsgemeinschaft der Frauen- und Geschlechterforschungseinrichtungen an Berliner Hochschulen (afg) gemeinsam mit der Landeskonferenz der Frauenbeauftragten an Berliner Hochschulen (LaKoF) und dem Deutschen Akademikerinnenbund (DAB) eine Festveranstaltung, die vom Regierenden Bürgermeister der Stadt und mehreren Senatsverwaltungen sowie der Überparteilichen Fraueninitiative Berlin unterstützt wurde.

In ihrer Begrüßungsrede hob die stellvertretende Vorsitzende der afg, Dorothea
Schmidt, hervor, dass die Akteurinnen der ersten Frauenbewegung in Deutschland jahrzehntelang um das Recht auf Bildung und Erwerbsarbeit und um das Recht zu wählen kämpfen mussten - und dass sie dies auf sehr unterschiedliche Weise taten. Neben Einzelkämpferinnen wie Hedwig Dohm, die mit spitzer Feder und loser Zunge die bürgerlichen Freiheitsrechte auch für Frauen einforderten, setzten sich bürgerliche Frauen um Helene Lange und Gertrud Bäumer ganz praktisch für die Frauenbildung ein, indem sie Mädchen unterrichteten. Für die proletarische Frauenbewegung um Clara Zetkin und Rosa Luxemburg war im Unterschied dazu das Recht auf Bildung nur einer der mög- 